\title{
Complete nucleotide sequence of the prophage VT2-Sakai carrying the verotoxin 2 genes of the enterohemorrhagic Escherichia coli 0157:H7 derived from the Sakai outbreak
}

\author{
Kozo Makino, ${ }^{1, *}$, Katsushi Yokoyama ${ }^{1}$, Yoshino Kubota ${ }^{1}$, Chikako H. Yutsudo ${ }^{1}$, \\ Sigenobu Kimura ${ }^{1}$, Ken Kurokawa ${ }^{2}$, Kazuo Ishii ${ }^{3}$, Masahiro Hattori ${ }^{3,7}$, \\ Ichiro Tatsuno ${ }^{4}$, Hiroyuki Abe ${ }^{4}$, Tetsuya Iida ${ }^{5}$, Koichiro Yamamoto ${ }^{5}$, \\ Makoto Onishi ${ }^{6}$, Tetsuya Hayashi ${ }^{6}$, Teruo Yasunaga ${ }^{2}$, Takeshi Honda ${ }^{5}$, \\ Chihiro Sasakawa ${ }^{4}$, and Hideo Shinagawa ${ }^{1,8}$ \\ ${ }^{1}$ Department of Molecular Microbiology, Research Institute for Microbial Diseases, \\ Osaka University, Suita, Osaka 565-0871 \\ ${ }^{2}$ Genome Information Research Center, Osaka University, Suita, Osaka 565-0871 \\ ${ }^{3}$ Human Genome Research Group, RIKEN Genomic Science Center, \\ c/o Kitasato University, Sagamihara, Kanagawa 228-8555 \\ ${ }^{4}$ Department of Bacteriology, Institute of Medical Science, The University of Tokyo, \\ Shiroganedai, Minatoku, Tokyo 108-8639 \\ ${ }^{5}$ Department of Bacterial Infections, Research Institute for Microbial Diseases, \\ Osaka University, Osaka 565-0871 \\ ${ }^{6}$ Department of Bacteriology, School of Medicine, Shinshu University, Matsumoto 390-8621 \\ ${ }^{7}$ Human Genome Center, Institute of Medical Science, The University of Tokyo, \\ Shiroganedai, Minatoku, Tokyo 108-8639, and \\ ${ }^{8}$ Molecular Genetics Laboratory, Osaka University, Suita, Osaka 565-0871
}

(Received 22 September, accepted 23 October)

The enterohemorrhagic Escherichia coli (EHEC) O157:H7 strain RIMD 0509952, derived from an outbreak in Sakai city, Japan, in 1996, produces two kinds of verotoxins, VT1 and VT2, encoded by the stx 1 and stx2 genes. In the EHEC strains, as well as in other VT-producing $E$. coli strains, the toxins are encoded by lysogenic bacteriophages. The EHEC O157:H7 strain RIMD 0509952 did not produce plaque-forming phage particles upon inducing treatments. We have determined the complete nucleotide sequence of a prophage, VT2-Sakai, carrying the stx $2 A$ and stx $2 B$ genes on the chromosome, and presumed the putative functions of the encoded proteins and the cis-acting DNA elements based on sequence homology data. To our surprise, the sequences in the regions of VT2-Sakai corresponding to the early gene regulators and replication proteins, and the DNA sequences recognized by the regulators share very limited homology to those of the VT2-encoding 933W phage carried by the EHEC O157:H7 strain EDL933 reported by Plunkett et al. (J. Bacteriol., p1767-1778, 181, 1999), although the sequences corresponding to the structural components are almost identical. These data suggest that these two phages were derived from a common ancestral phage and that either or both of them underwent multiple genetic rearrangements. An IS629 insertion was found downstream of the stx $2 B$ gene and upstream of the lysis gene $S$, and this might be responsible for the absence of plaque-forming activity in the lysate obtained after inducing treatments.

\section{INTRODUCTION}

Enterohemorrhagic Escherichia coli (EHEC) is defined

* Corresponding author. as $E$. coli that produces one or more forms of Shiga toxin (Stx) and factors for attaching-and-effacing lesions on the host epithelial cells, and carries a characteristic large plasmid (Levine, 1987). EHEC causes not only hemorrhagic 
colitis, but also serious complications such as hemolytic uremic syndrome (HUS) and thrombotic thrombocytopenic purpura, sometimes resulting in death (Cohen and Giannella, 1991; Griffin and Tauxe, 1991). Among EHEC strains, the $E$. coli serotype O157:H7 is the most common one, and was first recognized in 1982 during outbreaks of bloody diarrhea in Oregon and Michigan, USA (Wells et al., 1983; Riley et al., 1983).

In Japan, several quite large outbreaks of EHEC O157:H7 occurred in 1996 (Watanabe et al., 1996). The biggest outbreak occurred in Sakai city, Osaka, where more than 6,000 patients suffered from diarrhea, hemorrhagic colitis and HUS. In this outbreak, approximately 1,000 patients were hospitalized for their severe gastrointestinal symptoms, and about 100 cases were complicated with HUS, resulting in 3 deaths.

We have previously determined the complete DNA sequences of the large virulence plasmid pO157 and the small plasmid pOSAK1 found in an EHEC O157 strain (RIMD 0509952) isolated from a typical case in the Sakai outbreak (Makino et al., 1998). pO157 possessed many pathogenic genes, such as $h l y A$, katP, espP and toxB, encoding EHEC hemolysin, catalase-peroxidase, serine protease, and a protein homologous to ToxinB of Clostridium difficile, respectively. It carried chimeric systems of replication and plasmid maintenance similar to those of R100 plasmid and F-factor. The strain RIMD 0509952 produces Shiga toxins, Stx1 and Stx2, encoded by two prophage-like regions, to which we named VT1Sakai and VT2-Sakai, respectively (unpublished data). When repeated efforts to detect phage plaque formation with the mitomycinC-induced Sakai strain were carried out with an indicator $E$. coli K-12 strain such as C600, no plaques were detected, indicating that the prophages carrying the stx 1 and stx 2 genes of this strain are defective for forming plaques. On the other hand, Matsushiro et al. (1999) reported that another O157:H7 Sakai strain RIMD 0509894 produced plaque-forming phages VT1-Sa and VT2-Sa, carrying the stx 1 and stx2 genes, respectively.

Here we report the complete DNA sequence of the prophage VT2-Sakai on the chromosome of the O157:H7 Sakai strain RIMD 0509952. This project is part of an ongoing effort to sequence the entire genome of the EHEC O157:H7 strain derived from the Sakai outbreak. Since Plunkett et al. (1999) published the complete DNA sequence of $933 \mathrm{~W}$ phage harboring the stx 2 genes of EHEC O157:H7 strain EDL933 (during our preparation of this manuscript), we compared the sequence between VT2Sakai and 933W and found substantial differences.

\section{MATERIALS AND METHODS}

Construction of cosmid- and lambda-based libraries of 0157 genomic DNA. The EHEC O157:H7 (RIMD 0509952) cells were grown as described previously
(Makino et al., 1998) and the whole genomic DNA was prepared by the standard method (Silhavy et al., 1984). The DNA was partially digested with Sau3A I restriction enzyme, treated with bacterial alkaline phosphatase to prevent multiple inserts, and ligated with SuperCos1 or lambda EMBL3 pre-digested with BamH I (Stratagene). Gigapack III XL packaging extracts (Stratagene) were used to package both the recombinant cosmids and lambda phages following the protocols of the supplier.

Isolation of cosmid and lambda phage clones carrying VT2-Sakai DNA, and filling the gap between the contigs by PCR. The stx2 DNA fragment (Fig. 1, probe A) used as a probe for colony and plaque hybridization was amplified by PCR using a TaKaRa Ex Taq kit (Takara Shuzo) with primers EVS1/2 (Takara Shuzo). The fragment was separated by conventional gel electrophoresis, and purified by using a QIAquick Gel Extraction kit (QIAGEN). An AlkPhos Direct kit (Amersham Life Science) was used to detect positive clones in hybridization experiments. To isolate the lambda clones which contain distant regions, such as the L31E09 clone (Fig. 1A), the appropriate primers were designed to make the probes $\mathrm{B}$ and $\mathrm{C}$ by PCR based on the sequence of the cosmid clone C49E05 ( Fig. 1A), and then probes B and C were used for plaque hybridization. In the case of the lambda clones, the inserted DNA fragments in the recombinant phages were amplified by PCR using a TaKaRa LA Taq kit (Takara Shuzo) with two primers flanking the cloning site: primer EMBL-1, (5'-ACAGTCGGTGGTCCGGCAGTACAATGGATTACC-3') and primer EMBL-2, (5'GCAACCTGCAACGTATTGAGCGCAAGAATCAGC-3'). The PCR products were treated with exonuclease I and shrimp alkaline phosphatase to remove excess dNTPs and primers (Amersham). To obtain the DNA fragment that fills the gap (Fig. 1A), primers A and B were used for PCR, and the PCR products were similarly treated with exonuclease I and shrimp alkaline phosphatase. These DNA fragments were used for shotgun cloning as described below.

Preparation of DNA templates for sequencing. Ten micrograms of the DNA fragments amplified by PCR or cosmid DNA were sonicated for $30 \mathrm{sec}$ with a sonic disrupter. The sonicated DNA was blunt-ended using a DNA blunting kit (Takara Shuzo) and fractionated by $6 \%$ polyacrylamide gel electrophoresis. A gel slice containing DNA fragments of $1 \sim 2 \mathrm{~kb}$ in length was excised from the gel, and DNA was eluted by immersing the gel in the MG elution buffer [500 mM ammonium acetate, $10 \mathrm{mM}$ magnesium acetate, $1 \mathrm{mM}$ EDTA, $0.1 \%$ (w/v) SDS] overnight at $37^{\circ} \mathrm{C}$. The recovered DNA was ligated to the Sma I site of vector M13mp18 DNA (Yanisch-Perron et al., 1985), which had been pretreated with bacterial alkaline phosphatase, and introduced into $E$. coli DH11S com- 


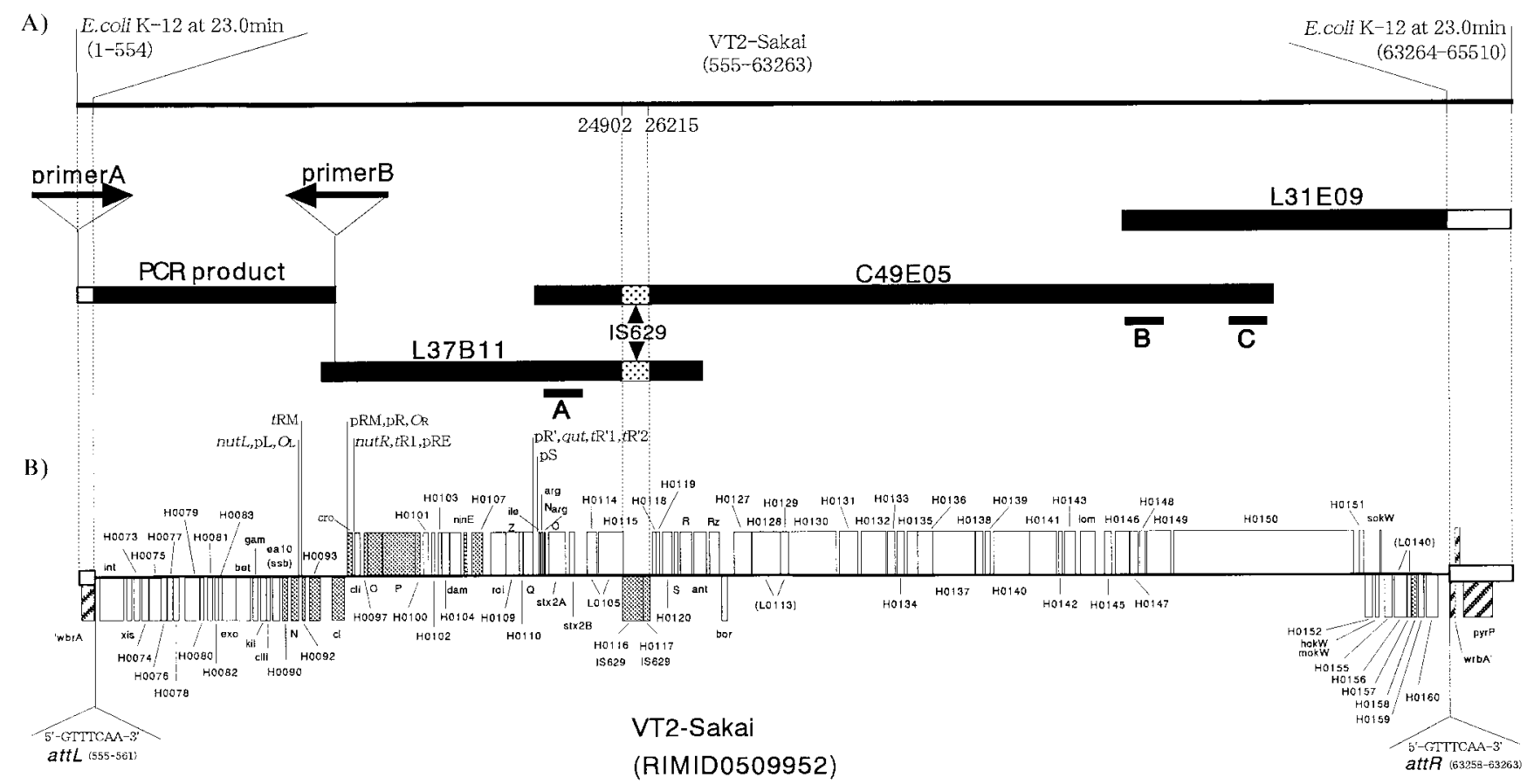

Fig. 1. Schematic illustration of the prophage VT2-Sakai. A) The location of VT2-Sakai on the chromosome is shown relative to the chromosome map of K-12 strain. The thick bars indicate the extents of the DNA fragments obtained from the lambda and cosmid clones, and by PCR amplification. To obtain clones L37B11 and C49E05, probe A was used for plaque or colony hybridization. To obtain L31E09, probes B and C were used for plaque hybridization. To fill the gap by PCR in the prophage region, two primers (A and B) were used. Open bars indicate regions corresponding to the K-12 chromosome. Black bars indicate regions corresponding to the prophage VT2-Sakai. Dotted bars indicate the IS629 region. The nucleotide numbers are counted from the left end of the PCR product. B) The predicted ORFs of VT2-Sakai are illustrated. Hatched boxes indicate ORFs also found in the K-12 genome. White and shaded boxes are ORFs encoded by the prophage VT2-Sakai. Shaded boxes are ORFs which have no or only limited homology to 933W. ORFs encoded by IS629 are also shaded. The boxes above and below the horizontal line are the OFRs with rightward and leftward transcription directions, respectively.

petent cells (Raleigh et al., 1988; Lin et al., 1992) with an electroporator. The cells were plated with LB soft agar containing X-gal $(100 \mu \mathrm{g} / \mathrm{ml})$, isopropyl $\beta$-D-thiogalactopyranoside $(1 \mathrm{mM})$ and indicator cells to screen the transfectants. The isolated plaques were inoculated into 100-fold diluted suspensions of overnight cell cultures and grown for $3 \mathrm{~h}$ at $37^{\circ} \mathrm{C}$, and the cultures were used as templates for PCR. The DNA inserts in the phage clones were amplified by PCR using a TaKaRa Ex Taq kit (Takara Shuzo) with two primers flanking the cloning site: primer 1, (5'-TCCGGCTCGTATGTTGTGTGGA-3') and primer 2, (5'-GTGCTGCAAGGCGATTAAGTTGG-3'). The PCR products were treated with a PCR product presequencing kit containing exonuclease I and shrimp alkaline phosphatase (Amersham).

DNA sequencing, sequence assembly and data analysis. The sequencing reactions were performed by using a Dye Primer Cycle Sequencing FS Ready Reaction Kit and a thermal cycler, GENEAMP PCR SYSTEM 9600 (Perkin-Elmer). DNA sequences were analyzed with an ABI PRISM 373XL DNA sequencer (Perkin-Elmer). Sequences were assembled by using the Factura 2.0
Feature Identification Software (Perkin-Elmer) and Autoassembler DNA Sequence Assembly Software Version 2.0 (Perkin-Elmer). To complete the sequencing of the entire prophage, 493 shotgun clones for the cosmid clone C49E05, 556 for the lambda clones L37B11 and L31E09, and 205 for the gap-filling PCR product were sequenced by using $-21 \mathrm{M} 13$ or/and M13Rev primers (PerkinElmer)(Fig. 1A).

The ORF Finder (from Other NCBI Resources) was used to identify potential open reading frames (ORFs). Each ORF was searched for in the sequence database using WWW BLASTP 2.0.8 (Jan/05/1999 version) (Altschul et al., 1997).

Nucleotide sequence accession number. The nucleotide sequence data reported in this paper will appear in the DDBJ/EMBL/GenBank nucleotide sequence databases with accession number AP000422.

\section{RESULTS}

Sequence of VT2-Sakai and its surrounding regions. To analyze the unique DNA sequence to the O157 strain 
RIMD 0509952, we determined the entire sequence of the prophage VT2-Sakai carrying the stx2A and stx2B genes after making cosmid and lambda libraries with the genomic DNA. We screened the libraries by colony or plaque hybridization using astx2 DNA fragment as a probe, and isolated clones that contained the stx 2 genes. One of them derived from the lambda library and another from the cosmid library were designated L37B11 and C49E05, respectively (Fig. 1A). The complete nucleotide sequences of the L37B11 and C49E05 clones were determined by the shotgun method as described in Materials and Methods. To clone lambda phages carrying the remaining regions, we screened the lambda library again by hybridization, using the probes $\mathrm{B}$ and $\mathrm{C}$ (Fig. 1A). The phages which hybridized with the two probes were partially sequenced, and one of the phages carried a sequence identical to that of a region of the K-12 genome, and was named L31E09 (Fig. 1A). Comparison of the complete nucleotide sequence of the insert of the L31E09 clone and that of the overlapping region of the K-12 genome revealed that the lambda clone carried the O157-specific region and the region corresponding to that of K-12 genome at $23 \mathrm{~min}$ (Fig. 1A) (Blattner et al., 1997). The end of the homologous region to the K-12 genome was located within the $w r b A$ gene, which encodes the Trp repressor binding protein, WrbA. It has been proposed that the WrbA protein functions as an accessory protein in blocking TrpR-specific transcriptional repression (Yang et al., 1993). To fill the gap, we constructed primers that were specific for $w r b A$ (Blattner et al., 1997) and the end of L37B11 based on its sequence information (Fig. 1A, primers $\mathrm{A}$ and $\mathrm{B}$ ). The DNA fragment containing the gap region was amplified by PCR with the genomic DNA extracted from the O157:H7 cells, and its sequence was determined by the shotgun method (Fig. 1A). The combination of the sequence of the PCR product and all the sequence data described above indicated that the $0157: H 7$-specific region was about $62.7-\mathrm{kb}$ long and was integrated into the $w r b A$ gene of the K-12 genome (Fig. 1).

Identification of the attachment site of VT2-Sakai. The sequence of the VT2-Sakai prophage is flanked by two copies of a 7-bp direct repeat sequence (GTTTCAA) present only once in the $w r b A$ gene of K-12 (Fig. 1B). Like lambda phage, many temperate bacteriophages integrate into their host genomes by a site-specific recombination between the phage and bacterial attachment sites, $a t t P$ and $a t t B$. As a result, the integration generates two copies of a direct repeat ( $a t t L$ and $a t t R$ ) flanking the integrated linear prophage genome. From these reasons, we concluded that the core att $L$ and $a t t R$ sequences were located at positions 555 to 561 (attL) and positions 63258 to 63263 (attR), respectively (Fig. 1B). The total length of the VT2-Sakai prophage with the two attachment sites was $62708 \mathrm{bp}$. The $\mathrm{G}+\mathrm{C}$ content of the VT2-Sakai prophage was $49.9 \%$, which is slightly lower than that of the K-12 genome (50.8\%).

IS element. The $w r b A$ gene, whose sequence was identical to that of the gene from the K-12 strain was split by integration of VT2-Sakai, and the junctions were identified at two regions (1-554 and 63264-65550) (Fig. 1A). We found an IS element at positions 24899 to 26209 that was very similar to the IS629 derived from Shigella sonnei (96.0\% identity) (Fig. 1) (Matsutani and Ohtsubo, 1990). A 4-base direct repeat at the insertion site of IS629 was identified and it split an $\mathrm{ORF}(\mathrm{H} 0115$; see below) whose function could not be identified from the homology search. The IS629 insertion is likely to be responsible for the deficiency in plaque-forming ability, although we do not know whether the deficiency is due to the insertion inactivation of an essential gene or the reduction of the transcription of downstream genes, such as the $S$ and $R$ genes, required for the lysis of the host cells.

Comparison between the DNA sequences of VT2Sakai and 933W. Plunkett et al. (1999) have recently determined the complete sequence the stx2-encoding phage 933W derived from the O157:H7 strain EDL933. We compared the DNA sequences and ORFs of the two phages, both of which carry the stx 2 genes. The integration site of VT2-Sakai on the chromosome is exactly the same as that of 933W. VT2-Sakai and 933W share very similar DNA sequences in most areas of the genomes, with only minor sequence differences and small gaps. Notable differences were found in eight bocks where there existed little sequence similarity, including the one created by the IS629 insertion (Fig. 2). These regions included the regulator genes for the early gene expression, the genes for replication initiation, and the recognition sequences for the regulators (see below). Except for IS629, they were located between nucleotides 9137 and 18975, where six small nonidentical blocks were flanked by highly conserved sequences. Homology search analysis of the DNA sequence from nucleotide 8000 to 19500 revealed that this region is highly chimeric (Fig. 3). The DNA sequences from 8000 to 9137 , from 10015 to 10084, and from 11459 to 11865 are very similar to those of lambda phage (Sanger et al., 1982). The DNA sequence from 9838 to 9888 is homologous to that of $\phi 21$ phage (Franklin, 1985). The DNA sequences from 12577 to 15178 and from 18774 to 18844 are almost identical to those of HK022 phage (Oberto et al., 1989; Oberto et al., 1994; Clerget and Boccard, 1996). The DNA sequences corresponding to those from 17505 to 17700 , from 18384 to 18740 , and from 18962 to 19100 are very similar to those of $\phi 21$ (Kroeger, 1999), P22 (Sauer et al., 1983), and H-19B phages (Neely and Friedman, 1998a; Neely and Friedman, 1998b), respectively. These results suggest that many rounds of recombination with these and other unknown phages gen- 


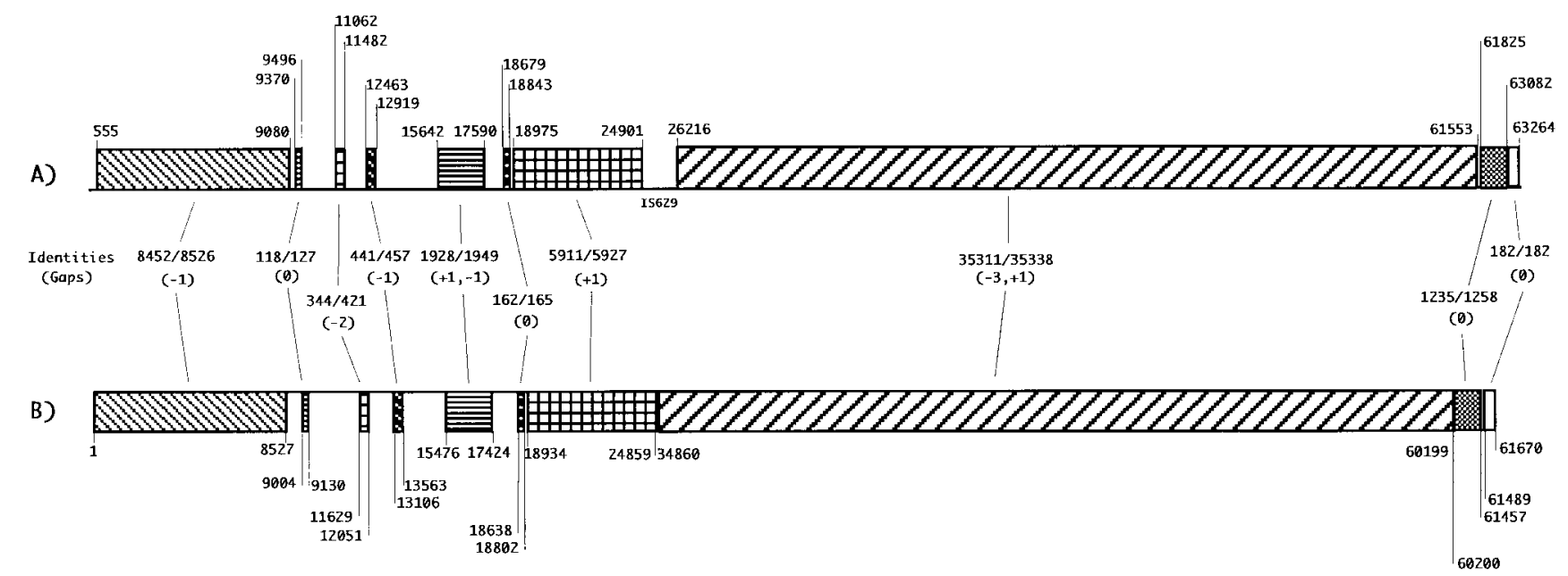

Fig. 2. Schematic drawing of the regions nearly identical in VT2-Sakai (A) and 933W (B). Homologous regions are boxed. Numbers of identical bases per total number of bases compared are shown. The numbers with minus and plus signs in parentheses indicate the numbers of bases missing or added in the VT2-Sakai sequence as compared with the 933W, respectively.

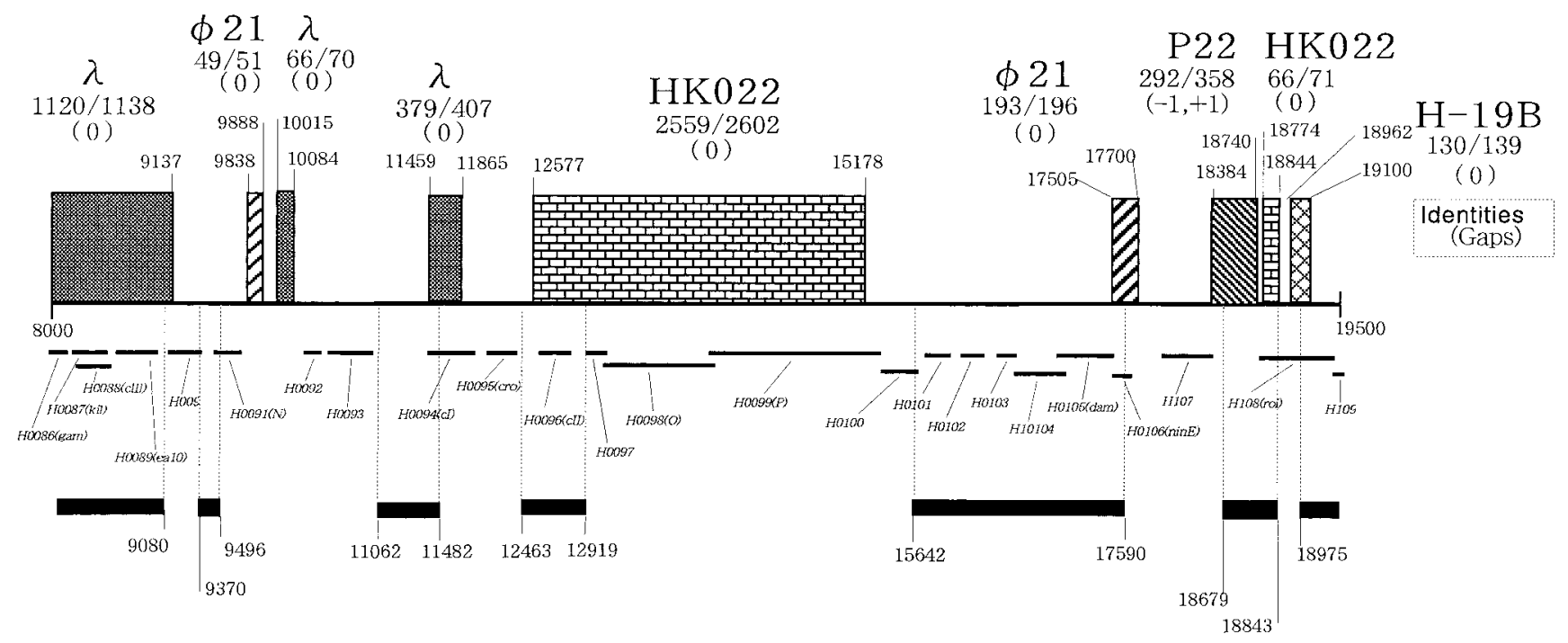

Fig. 3. DNA sequence in the region of VT2-Sakai from nucleotide 8000 to 19500, which contains several blocks of sequences different from those of 933W. Black bars indicate the regions homologous to those of 933W. The regions homologous to other phages are shown by the boxes filled with various patterns specific to phages whose names are shown above. The numbers of identical bases per total number of bases compared are shown. The numbers in parentheses indicate the numbers of bases missing (-) or added (+) in the VT2Sakai sequence as compared with those of other phages.

erated VT2-Sakai. It should be noted that the regions described above encode the immunity proteins, including $\mathrm{CI}$ and Cro proteins, and their recognition sequences and therefore VT2-Sakai is likely to have immunity specificity different from that of $933 \mathrm{~W}$.

\section{Descriptions of selected ORFs based on homology} search. We listed ORFs longer than 100 amino acids (aa) in Table 1 and used them for homology search analysis against the NCBI's non-redundant amino acid sequence database, $\mathrm{nr}$ (Jan/05/99 version). When ORFs shorter than 100 aa were found to be homologous to any known gene product, they were also noted and listed in Table 1. Computational analyses of the entire nucleotide sequence identified $93 \mathrm{ORFs}$ and three putative tRNA genes (Table 1), which are illustrated in Fig. 1B. ORFs in the VT2-Sakai prophage are labeled H0071 to H0160, from left to right in Fig. 1B. Genes with functional assignments are named, and the names of the equivalent lambda genes or $E$. coli K-12 genes were employed when possible.

The VT2-Sakai prophage is very similar to the bacteriophage 933W (Plunkett et al., 1999) carrying VT2 (stx2) genes at the nucleotide sequence and gene organization 
Table 1. Genes of VT2-Sakai

\begin{tabular}{|c|c|c|c|c|c|c|c|}
\hline Label & Gene & From & To & Strand $^{\mathrm{a}}$ & $\begin{array}{l}\text { Length of } \\
\text { product } \\
\text { (amino } \\
\text { acids) }\end{array}$ & $\begin{array}{l}\text { Homologous } \\
\text { ORFs in } \\
933 \mathrm{~W}\end{array}$ & Note \\
\hline & 'wbrA & 8 & $<561$ & - & & & truncated wbrA of E.coli K-12 \\
\hline & attL & 555 & 561 & & & & attL core \\
\hline H0071 & int & 583 & 1917 & - & 444 & L0061 & integrase \\
\hline H0072 & xis & 1946 & 2245 & - & 99 & L0062 & excisionase \\
\hline H0073 & & 2316 & 2627 & - & 103 & L0063 & \\
\hline H0074 & & 2687 & 3052 & - & 121 & L0064 & \\
\hline H0075 & & 2964 & 3587 & - & 207 & L0065 & \\
\hline H0076 & & 3591 & 3878 & - & 95 & L0066 & \\
\hline H0077 & & 3880 & 4098 & - & 72 & L0067 & \\
\hline H0078 & & 4100 & 4387 & - & 93 & L0068 & \\
\hline H0079 & & 4657 & 5430 & - & 257 & L0069 & $\lambda$ Ea22 homolog \\
\hline H0080 & & 5427 & 5648 & - & 73 & L0141 & \\
\hline H0081 & & 5747 & 6028 & - & 93 & L0070 & $\lambda$ Orf-61 homolog \\
\hline H0082 & & 6039 & 6230 & - & 63 & L0071 & $\lambda$ Orf-63 homolog \\
\hline H0083 & & 6203 & 6391 & - & 62 & L0072 & $\lambda$ Orf-60a homolog \\
\hline H0084 & exo & 6382 & 7062 & - & 226 & L0073 & $\lambda$ Exo homolog; exonuclease \\
\hline H0085 & bet & 7059 & 7844 & - & 261 & L0074 & $\lambda$ Bet homolog; DNA annealing \\
\hline H0086 & gam & 7850 & 8146 & - & 98 & L0075 & $\lambda$ Gam homolog; inhibitor of RecBC \\
\hline H0087 & kil & 8221 & 8490 & - & 89 & L0076 & $\lambda$ Kil homolog; host killing \\
\hline H0088 & c III & 8333 & 8497 & - & 54 & L0077 & $\lambda$ CIII homolog; regulator for lysogenization \\
\hline H0089 & ea10 (ssb) & 8570 & 8938 & - & 122 & L0078 & $\lambda$ Ea10 homolog; ssDNA binding protein \\
\hline H0090 & & 9121 & 9372 & - & 83 & & \\
\hline H0091 & $\mathrm{N}$ & 9431 & 9703 & - & 90 & L0080 & phage $\mathrm{H}-19 \mathrm{~B} \mathrm{~N}$ homolog \\
\hline H0092 & & 10136 & 10258 & - & 40 & & \\
\hline H0093 & & 10432 & 10953 & - & 173 & & \\
\hline H0094 & c I & 11455 & 12150 & - & 231 & & $\lambda$ CI homolog; repressor for lysogeny \\
\hline H0095 & cro & 12226 & 12441 & + & 71 & & phage L C2 homolog; repressor for lytic growth \\
\hline H0096 & c II & 12583 & 12879 & + & 98 & L0087 & phage HK022 CII homolog; activator for $c$ I \\
\hline H0097 & & 12912 & 13058 & + & 48 & & phage HK022 Orf48 \\
\hline H0098 & $\mathrm{O}$ & 13051 & 13950 & + & 299 & & phage HK022 O homolog \\
\hline H0099 & $\mathrm{P}$ & 13940 & 15376 & + & 478 & & phage HK022 P homolog \\
\hline H0100 & & 15376 & 15645 & + & 89 & & \\
\hline H0101 & & 15716 & 15994 & + & 92 & & phage $933 \mathrm{~W}$ \\
\hline H0102 & & 16127 & 16342 & + & 71 & & phage $933 \mathrm{~W}$ \\
\hline H0103 & & 16353 & 16589 & + & 78 & & phage $933 \mathrm{~W}$ \\
\hline H0104 & & 16546 & 16992 & + & 148 & L0093 & $\lambda$ and P22 NinB Orf-146 homolog \\
\hline H0105 & dam & 16989 & 17516 & + & 175 & L0094 & phage HP1 and T1 Dam homolog; DNA adenine methylase \\
\hline H0106 & $\operatorname{nin} E$ & 17513 & 17695 & + & 60 & & phage 21 ninE homolog \\
\hline H0107 & & 17970 & 18485 & + & 171 & & phage TP901-1 \\
\hline H0108 & roi & 18768 & 19496 & + & 242 & L0096 & phage H-19B Roi homolog \\
\hline H0109 & & 19496 & 20101 & + & 201 & L0097 & $\lambda$ NinG Orf-204 homolog \\
\hline H0110 & & 20098 & 20292 & + & 64 & L0098 & $\lambda$ NinH Orf-68 homolog \\
\hline \multirow[t]{10}{*}{ H0111 } & $\mathrm{Q}$ & 20285 & 20719 & + & 157 & L0099 & phage H-19B Q homolog \\
\hline & & 20707 & 20734 & + & & & $\mathrm{pR}$; putative late promoter \\
\hline & & 20748 & 20755 & + & & & qut; putative Q-utilization \\
\hline & & 20767 & 20789 & + & & & tR'1; possible rho-independent terminator \\
\hline & & 20970 & 20998 & + & & & tR'2; possible rho-independent terminator \\
\hline & & 21123 & 21151 & + & & & pS; putative tRNA promoter \\
\hline & ileZ & 21161 & 21236 & + & & L0100 & tRNA-Ile \\
\hline & $\operatorname{argN}$ & 21246 & 21322 & + & & L0101 & tRNA-Arg \\
\hline & $\operatorname{argO}$ & 21336 & 21412 & + & & L0102 & tRNA-Arg \\
\hline & & 21426 & 21449 & + & & & tS;possible rho-independent terminator \\
\hline H0112 & stx2A & 21503 & 22462 & + & 319 & L0103 & VT2 subunit A \\
\hline H0113 & stx2B & 22474 & 22743 & + & 89 & L0104 & VT2 subunit B \\
\hline H0114 & & 23230 & 23613 & + & 127 & L0105 & \\
\hline H0115 & & 23657 & 25135 & + & 492 & L0105 & \\
\hline H0116 & & 24942 & 25832 & - & 296 & & IS629; transposase \\
\hline H0117 & & 25829 & 26155 & - & 108 & & IS629 \\
\hline
\end{tabular}




\begin{tabular}{|c|c|c|c|c|c|c|c|}
\hline Label & Gene & From & To & Strand $^{\mathrm{a}}$ & $\begin{array}{c}\text { Length of } \\
\text { product } \\
\text { (amino } \\
\text { acids) }\end{array}$ & $\begin{array}{c}\text { Homologous } \\
\text { ORFs in } \\
933 \mathrm{~W}\end{array}$ & Note \\
\hline H0118 & & 26479 & 26604 & + & 42 & & phage $933 \mathrm{~W}$ \\
\hline H0119 & & 26617 & 26796 & + & 59 & & phage $933 \mathrm{~W}$ \\
\hline H0120 & & 26837 & 27109 & + & 90 & L0106 & \\
\hline H0121 & $\mathrm{S}$ & 27186 & 27401 & + & 71 & L0107 & phage H-19B and Qin S homolog \\
\hline H0122 & $\mathrm{R}$ & 27406 & 27939 & + & 177 & L0108 & phage Qin R homolog \\
\hline H0123 & ant & 28210 & 28779 & + & 189 & L0109 & phage P22 Ant homolog \\
\hline H0124 & $\mathrm{Rz}$ & 28933 & 29397 & + & 154 & L0110 & $\lambda$ Rz homolog \\
\hline H0125 & Rz1 & 29153 & 29338 & + & 61 & L0143 & $\lambda$ Rz1 homolog \\
\hline H0126 & bor & 29429 & 29722 & - & 97 & L0111 & $\lambda$ Bor homolog \\
\hline H0127 & & 30131 & 30937 & + & 268 & L0112 & \\
\hline H0128 & & 30918 & 32267 & + & 449 & L0113 & \\
\hline H0129 & & 32207 & 32623 & + & 138 & L0113 & \\
\hline H0130 & & 32623 & 34767 & + & 714 & L0114 & \\
\hline H0131 & & 34925 & 35932 & + & 335 & L0115 & \\
\hline H0132 & & 35956 & 37170 & + & 404 & L0116 & \\
\hline H0133 & & 37226 & 37615 & + & 129 & L0117 & \\
\hline H0134 & & 37638 & 38126 & + & 162 & L0118 & \\
\hline H0135 & & 38110 & 38673 & + & 187 & L0119 & \\
\hline H0136 & & 38673 & 39323 & + & 216 & L0120 & \\
\hline H0137 & & 39320 & 41257 & + & 645 & L0121 & similar to tail fiber protein \\
\hline H0138 & & 41259 & 41528 & + & 129 & L0122 & \\
\hline H0139 & & 41575 & 41856 & + & 93 & L0123 & \\
\hline H0140 & & 42073 & 43776 & + & 567 & L0124 & \\
\hline H0141 & & 43773 & 45041 & + & 422 & L0125 & \\
\hline H0142 & & 45107 & 45334 & + & 75 & L0126 & \\
\hline H0143 & & 45340 & 45957 & + & 205 & L0127 & \\
\hline H0144 & lom & 46048 & 46782 & + & 244 & L0128 & $\lambda$ Lom homolog \\
\hline H0145 & & 47212 & 47613 & + & 133 & L0129 & \\
\hline H0146 & & 47707 & 48363 & + & 218 & L0130 & \\
\hline H0147 & & 48366 & 48812 & + & 148 & L0131 & \\
\hline H0148 & & 48822 & 49073 & + & 83 & L0132 & \\
\hline H0149 & & 49084 & 50349 & + & 421 & L0133 & \\
\hline H0150 & & 50380 & 58800 & + & 2806 & L0134 & \\
\hline H0151 & & 59083 & 59271 & + & 62 & L0135 & \\
\hline H0152 & & 59351 & 59695 & - & 114 & L0136 & similar to YgiW and YdeI of E. coli \\
\hline H0153 & hokW & 59815 & 59970 & - & 51 & L0137 & similar to Ref and Gef \\
\hline \multirow[t]{2}{*}{ H0154 } & mokW & 59815 & 60027 & - & 70 & L0144 & similar to Gef leader protein \\
\hline & sokW & 60019 & 60089 & + & & L0138 & antisense RNA \\
\hline H0155 & & 60270 & 60656 & - & 128 & L0139 & \\
\hline H0156 & & 60656 & 61315 & - & 219 & L0140 & \\
\hline H0157 & & 61336 & 61554 & - & 72 & L0140 & \\
\hline H0158 & & 61541 & 61825 & - & 94 & & \\
\hline H0159 & & 61822 & 62043 & - & 73 & L0141 & \\
\hline \multirow[t]{5}{*}{ H0160 } & & 62091 & 62720 & - & 209 & L0142 & \\
\hline & attR & 63258 & 63263 & + & & & attR core \\
\hline & wrbA' & $<63258$ & 63307 & - & & & truncated wbrA of E. coli K-12 \\
\hline & & 63613 & 63786 & + & & & Hypothetical protein of E. coli K-12 \\
\hline & pyrP & 63869 & 65197 & - & & & uracil transport protein of E. coli K-12 \\
\hline
\end{tabular}

a. A plus sign indicates the forward orientation of the gene and a minus sign indicates the reverse orientation of the gene.

levels (Fig. 2 and Table 1).

ORFs H0071 (Int) to H0089 (Ea10) are identical or very similar to the corresponding ORFs of 933W (L0061 to L0078)(Fig. 1B and Table 1). This region includes the homologs encoding the integrase that mediates the sitespecific recombination necessary for integration into the host genome and xis, encoding the excisionase that together with integrase mediates excision of the phage genome from the host genome upon induction (Weisberg and Landy, 1983). The region from H0084 to H0086 contains the exo, bet and gam genes highly homologous to the homologous recombination system of lambda (Smith, 1983). Downstream of this region, the kil and $c$ III genes, which are involved in host cell killing and establishment 
of lysogeny (Court and Oppenheim, 1983; Wulff and Rosenberg, 1983), respectively, are also very similar in sequence to the lambda homologs. ORFs H0090, 0092, 0093 and 0100 are not homologous to any known ORFs in the database. ORF H0091 is similar to the antitermination $\mathrm{N}$ proteins of bacteriophage $\mathrm{H}-19 \mathrm{~B}$ and $933 \mathrm{~W}$, although the similarities are relatively low (42 and 41\% identities, respectively) (Neely and Friedman, 1998a; Neely and Friedman, 1998b; Plunkett et al., 1999). ORF H0094 is the CI repressor of VT2-Sakai, which appears to be a hybrid of two species of repressors. The amino-terminal 96-amino acid sequence most closely resembles that of the repressor of bacteriophage L (56\% similarity) (Schicklmaier and Schmieger, 1997), while the rest of the sequence is almost identical to that of lambda (99\% identity) (Sanger et al., 1982). In the lambda repressor, the amino-terminal domain contains a helix-turn-helix motif for DNA binding that interacts with the operator sequences, while the carboxyl-terminal domain of the protein is involved in dimerization (Gussin et al., 1983). Although the CI of VT2-Sakai is not similar to that of 933W, the CI of 933W is a chimera of the repressors of two bacteriophages, HK022 and H-19B (Plunkett et al., 1999).

ORF H0095 is most similar to the Cro repressor of bacteriophage L ( $42 \%$ identity) (Schicklmaier and Schmieger, 1997). The similarities of the amino-terminal domain of CI described above and the Cro sequence to those of phage $\mathrm{L}$ are consistent with the facts that both $\mathrm{CI}$ and Cro recognize the same operator sequences, which has been shown in lambda (Gussin et al., 1983). ORF H0096 is similar to the CII proteins of HK022 (96\% identity) (Oberto et al., 1989; Oberto et al., 1994), 933W (95\% identity) (Plunkett et al., 1999), and H-19B (94\% identity) (Neely and Friedman, 1998a; Neely and Friedman, 1998b), respectively. ORF H0097 is identical to the hypothetical protein 48 of phage HK022(100\% identity) (Oberto et al., 1994) and similar to ORF48 of phage P22(85\% identity) (Backhaus and Petri, 1984). This ORF is not present in 933W. ORFs H0098 and H0099 are nearly identical to the replication proteins $\mathrm{O}$ (98\% identity) and $\mathrm{P}$ (98\% identity) of phage HK022 (Oberto et al., 1994), respectively, but they show no homologies to those of 933W. In the well-characterized lambda replication system, the origin of replication is located in the coding region of the $\mathrm{O}$ protein, in which there are four 20-bp direct repeat sequences that are recognized by the $\mathrm{O}$ protein to initiate the replication (Furth and Wickner, 1983). Since the replication origin of HK022 is not known, we looked for such sequences based on the analogy to lambda. We found two 30-bp direct repeat sequences (5'-TGTACCAGCAGATTACCAGCAAATTACCAC) at positions 13443 to 13472 and 13509 to 13538 in the coding region for the $\mathrm{O}$ protein of VT2-Sakai. Consequently, the VPADYQQITT sequence appears twice in the $\mathrm{O}$ protein. This kind of repeat peptide is not present in the $\mathrm{O}$ protein of lambda. Such repeat sequences of DNA and/or peptides may be related to the DNA-O protein interaction(s) required for the initiation of replication of VT2-Sakai. Although ORF H0100 is not similar to any known ORFs, it may correspond to the Ren protein involved in the reaction of Rex exclusion from the conserved relative position on the genome and its relative size. ORFs H0101 to H0103 were not noted in the $933 \mathrm{~W}$ sequence reported by Plunkett et al. (1999), but Schmidt et al. (1997) reported their existence in 933W (GenBank; Y10775) (Schmidt et al., 1997). The DNA sequence of this region in VT2-Sakai is identical to that in $933 \mathrm{~W}$ reported by Schmidt et al. (1997), and it differs from the one reported by Plunkett et al. (1999) by a 1-bp deletion in their sequence.

ORF H0104 and H0105 (Dam) are very similar to L0096 and L0097 of 933W (both 99\% identities) (Plunkett et al., 1999). H0105 is similar to a hypothetical adenine-specific DNA methylase of bacteriophage HP1 (38\% identity) (Esposito et al., 1996). It has been proposed that such a DNA modification is related to bacterial virulence (Heithoff et al., 1999).

ORF H0106 is almost identical to the to NinE proteins of bacteriophages 21 (98\% identity) (Kroeger, 1999), P22 (98\% identity) (Kroeger, 1994), H-19B (98\% identity) (Neely and Friedman, 1998a; Neely and Friedman, 1998b) and lambda (93\% identity) (Sanger et al., 1982), and very similar to that of 933W (85\% identity) (Schmidt et al., 1997). ORF H0107 is not present in $933 \mathrm{~W}$ and is most similar to a hypothetical protein of bacteriophage TP9011 (37\% identity) (Madsen and Hammer, 1998).

The ORFs from H0108 to H0160 are identical or very similar to the corresponding ORFs of 933W (L0096 to L0142)( Plunkett et al., 1999) except for the following: In VT2-Sakai, the ORF corresponding to L0105 of 933W is divided into two ORFs, H0114 and H0115, due to the addition of one G nucleotide at position 23589. Furthermore, the H0115 gene is fused to the IS629 sequence (Matsutani and Ohtsubo, 1990). ORFs H0116 and H0117 are the transposase and a hypothetical $12-\mathrm{kD}$ protein encoded by IS629, respectively. ORFs H0118 and H0119 were not noted in the $933 \mathrm{~W}$ sequence reported by Plunkett et al. (1999), but these ORFs were reported in the 933W sequence by Schmidt et al. (1997). L0140 of 933W is divided into ORFs H0156 and H0157 in VT2-Sakai due to a 1-bp deletion in the latter sequence. In VT2-Sakai, ORF H0158 is encoded by an additional 271-bp DNA sequence that is not present in 933W. Its sequence is not similar to any reported DNA sequence.

ORFs H0111, H0112, and H0113 correspond to Q, Stx2A and Stx2B. The existence of the transcriptional antiterminator $\mathrm{Q}$ and the related cis-acting DNA elements such as the late promoter $\mathrm{pR}$ ', qut, terminators $t \mathrm{R} 1$ ' and $t \mathrm{R} 2$ ', suggest that the late gene transcription is activated by the antitermination function of Q, which has been well studied in the lambda system (Friedman and Court, 1995; 
Ring and Roberts, 1994; Yarnell and Roberts, 1992). Therefore, the expression of the stx2 genes is likely to be positively regulated by $\mathrm{Q}$, like the expression of the downstream genes. This region is almost identical to that of 933W (Plunkett et al., 1999).

Promoters, operators, terminators and antiterminators in the immunity region. Between the $N$ and rexB genes of lambda phage lie three tandemly repeated operator sites, OL1, OL2 and OL3, each with inverted symmetry (Gussin et al., 1983). The operators recognized by $\mathrm{CI}$ and Cro proteins overlap and control the activities of the leftward promoter, pL (Gussin et al., 1983). The $\mathrm{pL}$ promoter is essential for the transcription of $N$, which is required for expression of the delayed early genes, such as $c$ III, int, $O, P$, and $Q$ in lambda (Gussin et al., 1983). In lambda, the region between the $N$ and rexB genes contains the nutL DNA sequence, whose transcript RNA signal is recognized by antiterminator protein $\mathrm{N}$, resulting in readthrough of the terminators $t \mathrm{~L} 1$ and $t \mathrm{~L} 2$ (Friedman and Gottesman, 1983). Moreover, this region contains the terminator $t \mathrm{RM}$, which blocks the trancription initiated from the pRM promoter (Gussin et al., 1983). The nucleotide sequence of the corresponding region of VT2Sakai is not similar to those of lamda, $933 \mathrm{~W}$, or other known bacteriophages, indicating that the recognition proteins N, CI and Cro are not similar to those of other known phages. As described in the previous section, the sequence similarities of $\mathrm{N}, \mathrm{CI}$ and Cro to those of other phages are relatively low, suggesting that these proteins recognize sequences different from those of other phages. By analogy to lambda, we searched by eyes for nucleotide sequences that might serve such functions in the region between the $N$ and $H 0092$ genes of VT2-Sakai. We found putative nutL, pL promoter, operators $(O \mathrm{~L} 1, O \mathrm{~L} 2$ and $O L 3)$, and terminator ( $t \mathrm{RM})$ sequences, as shown in Fig. 4.

Like in lambda phage, we also found three tandemly repeated operators, $O \mathrm{R} 1, O \mathrm{R} 2$ and $O \mathrm{R} 3$, each of which has an approximately inverted repeat structure, between the $c \mathrm{I}$ and cro genes of VT2-Sakai (Fig. 4). The sequence of

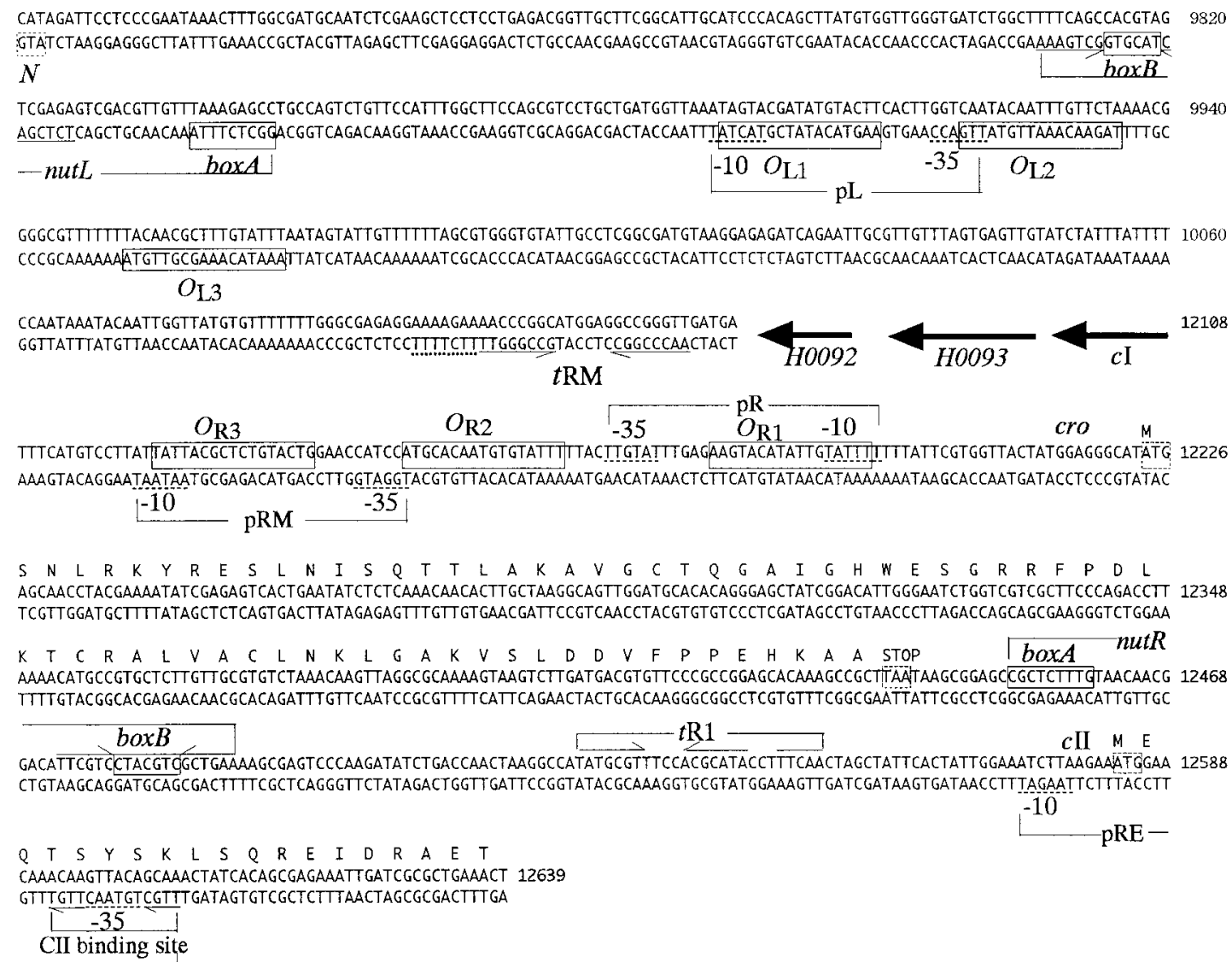

$\mathrm{pR}$

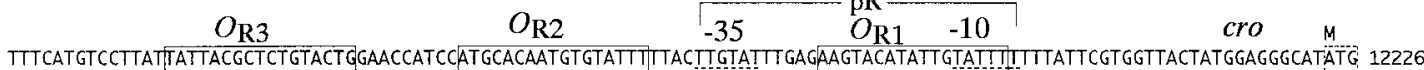

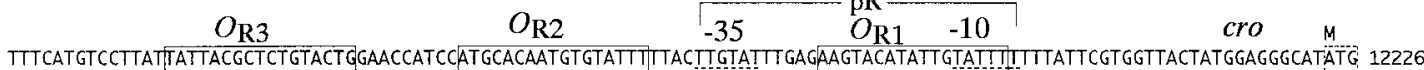
AAAGTACAGGAATAATAATGCGAGACATGACCTTGGTAGGTACGTGTTACACATAAAAATGAACATAAACTCTTCATGTATAACATAAAAAAATAAGCACCAATGATACCTCCCGTATAC $-10$ $-35$

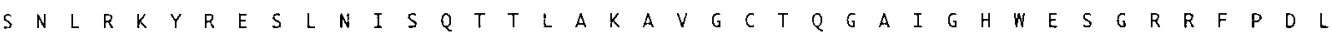
AGCAACCTACGAAAATATCGAGAGTCACTGAATATCTCTCAAACAACACTTGCTAAGGCAGTTGGATGCACACAGGGAGCTATCGGACATTGGGAATCTGGTCGTCGCTTCCCAGACCTT 12348 TCGTTGGATGCTTTTATAGCTCTCAGTGACTTATAGAGAGTTTGTTGTGAACGATTCCGTCAACCTACGTGTGTCCCTCGATAGCCTGTAACCCTTAGACCAGCAGCGAAGGGTCTGGAA

K T C R A L V A C L N K L G A K V S L D D V F P P E H K A A STOP AAAACATGCCGTGCTCTTGTTGCGTGTCTAAACAAGTTAGGCGCAAAAGTAAGTCTTGATGACGTGTTCCCGCCGGAGCACAAAGCCGCTITAÄTAAGCGGAGCCGCTCTTTGTAACAACG 12468 ITTTGTACGGCACGAGAACAACGCACAGATTTGTTCAATCCGCGTTT CATTCAGAACTACTGCACAAGGGCGGCCTCGTGTTTCGGCGAÄTTATTCGCCTCGGCGAGAAACATTGTTGC GaCATTCGTCCTACGT OGCTGAAAAGCGAGTCCCAAGATATCTGACCAACTAAGGCCATATGCGTITCCACGCATACCTTTCAACTAGCTATTCACTATTGGAAATCTTAAGAAMTGGAA 12588 CTGTAAGCAGGATGCAGCGACTTTTCGCTCAGGGTTCTATAGACTGGTTGATTCCGGTATACGCAAAGGTGCGTATGGAAAGTTGATCGATAAGTGATAACCTTTAGAATTCTTTACCTT$$
-10
$$

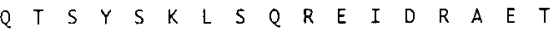
pRECAAACAAGTIACAGCAAACTATCACAGCGAGAAATTGATCGCGCTGAAACT 12639 GTTTGTTCAATGTCGTTTGATAGTGTCGCTCTTTAACTAGCGCGACTTTGA

$-35$

CII binding site

Fig. 4. Sequence of VT2-Sakai in the region between H0091 $(N)$ and H0096 $(c \mathrm{II})$. The nucleotide sequence is shown in the conventional orientation for lambdoid phages. The features described in the text are indicated. The two sets of the segments labeled "boxA "and "boxB "constitute the putative nutL and nutR loci, respectively; the pairs of inward-facing arrows indicate the inverted repeat sequences of boxB. The putative repressor-binding sites $O_{\mathrm{L} 1}, O_{\mathrm{L} 2}, O_{\mathrm{L} 3}, O_{\mathrm{R} 1}, O_{\mathrm{R} 2}$, and $O_{\mathrm{R} 3}$ are boxed, and the presumed -35 and -10 regions of the $\mathrm{pL}, \mathrm{pRM}, \mathrm{pR}$ and $\mathrm{pRE}$ promoters are underlined with broken lines. The regions labeled " $t \mathrm{RM}$ " and " $t \mathrm{R}$ " constitute the putative terminators for $\mathrm{pRM}$ and $\mathrm{pR}$, respectively. The CII-binding site indicated in the pRE promoter is located in the position corresponding to the -35 region. The three large arrows indicate the locations and directions of the H0O92, H0O93 and $c \mathrm{I}$ genes. 
this region is not similar to those of other phages. The operators, which may be recognized by CI and Cro of VT2Sakai, overlap the putative rightward promoter, $\mathrm{pR}$, as well as the putative leftward promoter, pRM. By analogy to lambda, the former may transcribe cro and other early genes of VT2-Sakai; the latter may be responsible for transcription of $c \mathrm{I}$ for maintaining the repressed state. The six operators described above give a consensus sequence with inverted symmetry (Fig. 5).

Lambda, P22 and $\phi 21$ contain cryptic promoters activated by their respective CII proteins (Wulff and Rosenberg, 1983; Wulff and Mahoney, 1987). They lack conserved -35 regions, but have the typical -10 regions. One such lambda promoter, pRE, is responsible for transcription of the $c \mathrm{I}$ gene after infection to establish lysogeny (Wulff and Rosenberg, 1983). It is located downstream of the cro gene. A search for the corresponding sequence in this region of VT2-Sakai revealed a sequence that shares the consensus TTGC repeat of the CII recognition sequence with lambda: TTGCN6TTGC (Fig. 4) (Wulff and Mahoney, 1987). Downstream of the putative recognition sequence, there is a reasonable -10 sequence. It is likely that these sequence elements constitute the pRE promoter of VT2-Sakai. Like in lamda phage, the promoter overlaps the coding sequence for CII. Also like in lambda, the putative antitermination signal, nutR, and the putative terminator, $t \mathrm{R} 1$, were found between the cro and $c \mathrm{II}$ (Fig. 4). Transcription antitermination in lambda requires the phage nut sites (Friedman and Gottesman, 1983). N protein attaches to RNA polymerase at the $n u t$ sites to form a termination-resistant complex (Friedman and Court, 1995; Das, 1992). The nut sites can be divided into a promoter-proximal sequence, boxA, conserved among several lambdoid phages, and boxB, a non-conserved region of hyphenated dyad symmetry (Friedman and Gottesman, 1983; Friedman et al., 1990). The variations in boxBs are related to the different $N$ genes and, in

\section{OL1 AAGTACATA TAGTACGA}

\section{OL2 TAGAACAAA \\ CAATACAA}

\author{
OL3 AAATACAAA \\ TACAACGC
}
OR1 AAGTACATA
AAATACAA
OR2 ATGCACAAT
AAATACAC

\section{OR3 TATTACGCT \\ CAGTACAG}

\section{CONSENSUS aAgTACAa}

Fig. 5. The putative recognition sequences (operators) of VT2Sakai. To obtain a consensus sequence for the repressor-binding site, the 6 sequences of $O_{\mathrm{L} 1}, O_{\mathrm{L} 2}, O_{\mathrm{L} 3}, O_{\mathrm{R} 1}, O_{\mathrm{R} 2}$, and $O_{\mathrm{R} 3}$ were broken near the midpoints to give 12 half-sites. The sequence of one member of each pair of half-sites was inverted with respect to the other, and the sequences of the top (5' to 3') strands of the half-sites were aligned with each other. A consensus sequence was obtained from the aligment of the 12 half-sites, and is indicated at the bottom.

part, account for their specificities (Friedman and Court, 1995; Das, 1992). Lambda carries two nearly identical nut sites: nutL, located between $\mathrm{pL}$ and $N$, and $n u t R$, which is downstream of cro (Friedman and Gottesman,

$\operatorname{box} A$

box $B$

$\begin{aligned} \text { VT2-Sakai } n u t R & \text { CGCTCTTTGtaacaacggacaTTCGTCTACGTCGCTGAA } \\ \text { VT2-Sakai } n u t L & \text { GGCTCTTTAaacaacgtcgacTCTCGACTACGTGGCTGAAA } \\ \text { lambda } n u t R & \text { CGCTCTTAcacattccaGCCCTGAAAAGGGC } \\ \text { lambda } n u t L & \text { CGCTCTTAaaaattaaGCCCTGAAGAGGGC } \\ 21 \text { nutR } & \text { TGCTCTTTAacagttctggccttTCACCTCTAACGGGTGG } \\ 21 \text { nutL } & \text { GGCTCTTTAacatcgacggacTCTCAACTAACCGTGAGA }\end{aligned}$

H-19B nutR CGCTATTTTCacaatggacattcgtcctacgICGCTGACAAGCGA

H-19B nutL CGCACTACTCaccagggcggtgatatacaacgattcgaatatgaatctacgGCGCTGACAAAGCGC

Fig. 6. Comparison of the sequences of the nut sites of VT2-Sakai with those of other lamboid phages. 
1983). Despite the lack of similarity of the DNA sequence in these regions between lambda and VT2-Sakai, both sequences carry putative nut sites at the corresponding positions (Fig. 4). In VT2-Sakai, the sequences of the boxA and boxB sites of nutR and nutL are similar to each other. The sequences of the boxAs are similar to those of the $n u t$ sites of other phages, while the sequences of the boxBs are unique to VT2-Sakai. This fact suggests that the N protein of VT2-Sakai probably specifically recognizes these $n u t$ sites (Fig. 6).

\section{DISCUSSION}

We have determined the complete nucleotide sequence of the prophage, VT2-Sakai carrying stx2 genes, of EHEC O157:H7 (RIMD 0509952). To our surprise, the DNA sequence of this prophage was different in several regions from that of a VT2 carrying phage $933 \mathrm{~W}$ which was isolated from another EHEC O157:H7 (EDL933), although the rest of the sequence were nearly identical. These two phages showed little homology in the sequences related to the expression of immediate early genes, which define the immunity, and therefore they should possess different immune specificities. In addition, VT2-Sakai carries an IS629, which may contribute to causing the defectiveness of this prophage.

It has been reported that transcription of the stx genes is induced by treatment with mitomycinC only when these genes are encoded by prophages (Takeda et al., 1979; Yee et al., 1993). Production of VT2 by EHEC O157:H7 (RIMD 0509952) was also induced by treatment with mitomycinC or with UV irradiation (unpublished data). During the lytic cycle, the late regulator protein, Q, counteracts the termination of transcription initiated from the late promoter $\mathrm{pR}$ ' using the qut sequence that partially overlaps the promoter. $933 \mathrm{~W}$ and $\mathrm{H}-19 \mathrm{~B}$ phages are known to have different immune specificities and homologous Q systems (Plunkett et al., 1999; Neely and Friedman, 1998a; Neely and Friedman, 1998b). The Q proteins of $\mathrm{H}-19 \mathrm{~B}$ and $933 \mathrm{~W}$ are exchangeable for the induction of each other's stx genes and late genes, including those for lysis and for head and tail proteins (Neely and Friedman, 1998a). Because the Q, pR' and qut sequences of VT2-Sakai are identical or similar to those of 933W and $\mathrm{H}-19 \mathrm{~B}$, the regulation of the expression of VT2 (Stx2) in VT2-Sakai may be similar to those reported (Plunkett et al., 1999; Neely and Friedman, 1998a; Neely and Friedman, 1998b).

VT2-Sakai has an IS629 insertion upstream of the genes that encode lysis proteins. Transcription initiated from the late promoter pR' may be attenuated or blocked by the insertion of IS629. As a result, sufficient amount of late proteins, including lysis proteins and structural proteins for the phage particle, to produce infective phage may not be produced. Although it is still unclear how toxins are released from bacteria to cause disease, it has been proposed that death of the bacteria with associated autolysis must occur for the release of toxins. It has also been proposed that expression of the lysis genes is an important factor for the release of Stx toxins. If this is true, since the lysis proteins in VT2-Sakai may not be produced due to the IS629 insertion, lysis proteins must be supplied in trans by other prophages after induction of lytic growth. It is possible that the lysis protein may be provided by some other prophage(s) lysogenized in the strain.

In the sequence data bases, there are only two cases of stx genes described as chromosomal gene sequences: one is Shigella dysenteriae (Kozlov et al., 1988) and the other is E. coli O111: $\mathrm{H}^{-}$(Paton et al., 1993). Although the entire DNA sequences with their surrounding regions are not available, there are common features in these cases: the existences of $I S$ elements and phage-like sequences near the toxin genes (Mizutani et al., 1999). These features are very similar to those of the VT2-Sakai prophage in the Sakai strain. These data suggest that the stx2 genes previously thought to be chromosomal genes will turn out to be encoded by prophages and insertion of an IS element might have been responsible for making them defectiveness. The ongoing genome project to analyze the genome of the Sakai strain has also revealed that more than ten phage-like sequences exist in the genome (unpublished data). The apparent chimeric nature of VT2Sakai might have resulted from multiple recombination events with other prophages residing in the genome.

Recently, Miyamoto et al. (1999) have published the complete sequence of the Stx2-converting phage VT2-Sa derived from another O157:H7 Sakai strain RIMD 0509894. The VT2-Sa phage does not carry the IS element and bor (H0126) gene that exist in VT2-Sakai, and has an amber mutation in the $c$ I gene.

We thank Drs. N. Ogasawara and T. Nakazawa for their valuable suggestions and support. This work was supported by a grant, "Research for the Future" Program 97L00101, from the Japan Society for the Promotion of Science.

\section{REFERENCES}

Altschul, S. F., Madden, T. L., Schaffer, A. A., Zhang, J., Zhang, Z., Miller, W., and Lipman, D. J. (1997) Gapped BLAST and PSI-BLAST: a new generation of protein database search programs. Nucleic Acids Res. 25, 3389-3402.

Backhaus, H., and Petri, J. B. (1984) Sequence analysis of a region from the early right operon in phage P22 including the replication genes 18 and 12 . Gene 32, 289-303.

Blattner, F. R., Plunkett, G. III, Bloch, C. A., Perna, N. T., Burland, V., Riley, M., Collado-Vides, J., Glasner, J. D., Rode, C. K., Mayhew, G. F., Gregor, J., Davis, N. W., Kirkpatrick, H. A., Goeden, M. A., Rose, D. J., Mau, B., and Shao, Y. (1997) The complete genome sequence of Escherichia coli K-12. Science 277, 1453-1474.

Clerget, M., and Boccard, F. (1996) Phage HK022 Roi protein inhibits phage lytic growth in Escherichia coli integration host 
factor mutant. J. Bacteriol. 178, 4077-4083.

Cohen, M. B., and Giannella, R. A. (1991) Hemorrhagic colitis associated with Escherichia coli O157:H7. Adv. Int. Med. 37, 173-195.

Court, D., and Oppenheim, A. B. (1983) Phage lambda's accessory genes. In: Lambda II (eds.: R. W. Hendrix, J. W. Roberts, F. W. Stahl, and R. A. Weisberg), pp. 251-277. Cold Spring Harbor Laboratory Press, Cold Spring Harbor, NY.

Das, A. (1992) How the phage lambda $N$ gene product suppresses transcription termination : communication of RNA polymerase with regulatory proteins mediated by signals in nascent RNA. J. Bacteriol. 174, 6711-6716.

Esposito, D., Fitzmaurice, W. P., Benjamin, R. C., Goodman, S. D., Waldman, A. S., and Scocca, J. J. (1996) The complete nucleotide sequence of bacteriophage HP1 DNA. Nucleic Acids Res. 24, 2360-2368.

Franklin, N. C. (1985) Conservation of genome form but not sequence in the transcription antitermination determinants of bacteriophage $\lambda, \phi 21$ and P22. J. Mol. Biol. 181, 75-84.

Friedman, D. I., and Gottesman, M. (1983) Lytic mode of lambda development. In: Lambda II (eds.: R. W. Hendrix, J. W. Roberts, F. W. Stahl, and R. A. Weisberg), pp. 21-51. Cold Spring Harbor Laboratory Press, Cold Spring Harbor, NY.

Friedman, D. I., Olson, E. R., Johnson, L. L., Alessi, D., and Craven, M. G. (1990) Transcription-dependent competition for a host factor: the function and optimal sequence of the phage $\lambda$ boxA transcription antitermination signal. Genes. Dev. 4, $2210-2222$.

Friedman, D. I., and Court, D. L. (1995) Transcription antitermination: the $\lambda$ paradigm updated. Mol. Microbiol. 18, 191200.

Furth, E. F., and Wickner, S. H. (1983) Lambda DNA reprication. In: Lambda II (eds.: R. W. Hendrix, J. W. Roberts, F. W. Stahl, and R. A. Weisberg), pp. 145-173. Cold Spring Harbor Laboratory Press, Cold Spring Harbor, NY.

Griffin, P. M., and Tauxe, R. V. (1991) The epidemiology of infections caused by Escherichia coli O157:H7, other enterohemorrhagic $E$. coli, and the associated hemolytic uremic syndrome. Epidemiologic Rev. 13, 60-98.

Gussin, G. N., Johnson, A. D., Pabo, C. O., and Sauer, R. T. (1983) Repressor and Cro protein: structure, function, and role of lysogenization. In: Lambda II (eds.: R. W. Hendrix, J. W. Roberts, F. W. Stahl, and R. A. Weisberg), pp. 93-121. Cold Spring Harbor Laboratory Press, Cold Spring Harbor, NY.

Heithoff, D. M., Sinsheimer, R. L., Low, D. A., and Mahan, M. J. (1999) An essential role for DNA adenine methylation in bacterial virulence. Science 284, 967-970.

Kozlov, Y. V., Kabishev, A. A., Lukyanov, E. V., and Bayev, A. A. (1988) The primary structure of the operons coding for Shigella dysenteriae toxin and temprate phage H30 shiga-like toxin. Gene 67, 213-221.

Kroeger, M. (1994) Direct submission to DDBJ/EMBL/GenBank databases. Accession no. X78401.

Kroeger, M. (1999) Direct submission to DDBJ/EMBL/GenBank databases. Accession no. AJ237660.

Levine, M. M. (1987) Escherichia coli that cause diarrhea: enterotoxigenic, enteropathogenic, enteroinvasine, enterohemorrhagic, and enteroadherent. J. Infect. Dis. 155, 377389.

Lin, J. J., Smith, M., Jessee, J., and Bloom, F. (1992) DH11S: an Escherichia coli strain for preparation of single-stranded DNA from phagemid vector. Biotechniques 12, 718-721.

Madsen, P. L., and Hammer, K. (1998) Temporal transcription of the lactococcal temperate phage TP901-1 and DNA sequence of the early promoter region. Microbiology 144, 2203-2215.

Makino, K., Ishii, K., Yasunaga, T., Hattori, M., Yokoyama, K.,
Yutsudo, C. H., Kubota, Y., Yamaichi, Y., Iida, T., Yamamoto, K., Honda, T., Han, C. G., Ohtsubo, E., Kasamatsu, M., Hayashi, T., Kuhara, S., and Shinagawa, H. (1998) Complete nucleotide sequences of $93-\mathrm{kb}$ and $3.3-\mathrm{kb}$ plasmids of an enterohemorrhagic Escherichia coli $\mathrm{O} 157: \mathrm{H} 7$ derived from Sakai outbreak. DNA Res. 5, 1-9.

Matsutani, S., and Ohtsubo, E. (1990) Complete sequence of IS629. Nucleic Acids Res. 18, 1899.

Matsushiro, A., Sato, K., Miyamoto, H., Yamamura, T., and Honda T. (1999) Induction of prophages of enterohemorrhagic Escherishia coli O157:H7 with norfloxacin. J. Bacteriol. 181, $2257-2260$.

Miyamoto, H., Nakai, W., Yajima, N., Fujibayashi, A., Higuchi, T., Sato, K., and Matsushiro, A. (1999) Sequence analysis of Stx2-converting phage VT2-Sa shows a great divergence in early regulation and replication regions. DNA Res. 6, 235240.

Mizutani, S., Nakazono, N., and Sugino, Y. (1999) The so-called chromosomal verotoxin genes are actually carried by defective prophage. DNA Res. 6, 141-143.

Neely, M. N., and Friedman, D. I. (1998a) Functional and genetic analysis of regulatory regions of coliphage $\mathrm{H}-19 \mathrm{~B}$ : location of shiga-like toxin and lysis genes suggest a role for phage functions in toxin release. Mol. Microbiol. 28, 1255-1267.

Neely, M. N., and Friedman, D. I. (1998b) Arrangement and functional identification of genes in the regulatory region of lambdoid phage H-19B, a carrier of a Shiga-like toxin. Gene 223, $105-113$.

Oberto, J., Weisberg, R. A., and Gottesman, M. E. (1989) Structure and function of the nun gene the immunity region of the lambdoid phage HK022. J. Mol. Biol. 207, 675-693.

Oberto, J., Bohmer Sloan, S., and Weisberg, R. A. (1994) A segment of the phage HK022 chromosome is a mosaic of other lambdoid chromosome. Nucleic Acids Res. 22, 354-356.

Paton, A. W., Paton, J. C., Goldwater, P. N., Heuzenroeder, M. W., and Manning, P. A. (1993) Sequence of variant Shigalike toxin type-I operon of Escherichia coli O111:H'. Gene 129, 87-92.

Plunkett, G. III, Rose, D. J., Durfee, T. J., and Blattner, F. R. (1999) Sequence of Shiga toxin 2 phage 933W from Escherichia coli $\mathrm{O} 157: \mathrm{H} 7$ : Shiga toxin as a phage late-gene product. J. Bacteriol. 181, 1767-1778.

Raleigh, E. A., Murray, N. E., Revel, H., Blumenthal, R. M., Westaway, D., Reith, A. D., Rigby, P. W. J., Elhai, J., and Hanahan, D. (1988) McrA and McrB restriction phenotypes of some $E$. coli strains and implications for gene cloning. Nucleic Acids Res. 16, 1563-1575.

Riley, L. W., Remis, R. S., Helgerson, S. D., McGee, H. B., Wells, J. G., Davis, B. R., Hebert, R. J., Olcott, E. S., Johnson, L. M., Hargrett, N. T., Blake, P. A., and Cohen, M. L. (1983) Hemorrhagic colitis associated with a rare Escherichia coli serotype. N. Engl. J. Med. 308, 681-685.

Ring, B. Z., and Roberts, J. W. (1994) Function of a nontranscribed DNA strand site in transcription elongation. Cell 78, 317324.

Sanger, F., Coulson, A. R., Hong, G. F., Hill, D. F., and Petersen, G. B. (1982) Nucleotide sequence of bacteriophage $\lambda$ DNA. J. Mol. Biol. 162, 729-773.

Sauer, R. T., Krovatin, W., DeAnda, J., Youderian, P., and Susskind, M. M. (1983) Primary structure of the ImmI immunity region of bacteriophage P22. J. Mol. Biol. 168, 699-713.

Schicklmaier, P., and Schmieger, H. (1997) Sequence comparison of the genes for immunity, DNA replication, and cell lysis of the P22-related Salmonella phages ES18 and L. Gene 195, 93-100. 
Schmidt, H., Scheef, J., Janetzki-Mittmann, C., Datz, M., and Karch, H. (1997) An ileX tRNA gene is located close to the Shiga toxin II operon in enterohemorrhagic Escherichia coli O157 and non-O157 strains. FEMS Microbiol. Lett. 149, 3944.

Silhavy, T. J., Berman, M. L., and Enquist, L. W. (1984) In: Experiments with gene fusions. Cold Spring Habor Laboratory Press, Cold Spring Harbor, NY.

Smith, G. R. (1983) General recombination. In: Lambda II (eds.: R. W. Hendrix, J. W. Roberts, F. W. Stahl, and R. A. Weisberg), pp. 175-209. Cold Spring Harbor Labolatory Press, Cold Spring Harbor, NY.

Takeda, Y., Okamoto, K., and Miyatani, T. (1979) MitomycinC stimulates production of a toxin in Shigella species that causes morphological changes in Chinese hamster ovary cells. Infect. Immun. 23, 178-180.

Watanabe, H., Wada, A., Inagaki, Y., Itoh, K., and Tamura, K. (1996) Outbreak of enterohaemorrhagic Escherichia coli O157:H7 infection by two different genotype strains in Japan, 1996. Lancet 348, 831-832.

Weisberg, R. A., and Landy, A. (1983) Site-specific recombination in phage lambda. In: Lambda II (eds.: R. W. Hendrix, J. W. Roberts, F. W. Stahl and R. A. Weisberg), pp. 211-250. Cold Spring Harbor Laboratory Press, Cold Spring Harbor, NY.

Wells, J. G., Davis, B. R., Wachsmuth, I. K., Riley, L. W., Remis,
R. S., Sokolow, R., and Morris, G. K. (1983) Laboratory investigation of hemorrhanic colitis outbreaks associated with a rare Escherichia coli serotype. J. Clin. Microbiol. 18, 512520.

Wulff, D. L., and Rosenberg, M. (1983) Establishment of repressor synthesis. In: Lambda II (eds.: R. W. Hendrix, J. W. Roberts, F. W. Stahl, and R. A. Weisberg), pp. 53-73. Cold Spring Harbor Laboratory Press, Cold Spring Harbor, NY.

Wulff, D. L., and Mahoney, M. E. (1987) Cross-specificities between $c I I$-like proteins and $p_{R E}$-like promoters of lambdoid bacteriophages. Genetics 115, 597-604.

Yang, W., Ni, L., and Somerville, R. L. (1993) A stationary-phase protein of Escherichia coli that affects the mode of assosiation between the trp repressor protein and operator-bearing DNA. Proc. Natl. Acad. Sci. USA 90, 5796-5800.

Yanisch-Perron, C., Vieira, J., and Messing, J. (1985) Improved M13 phage cloning vectors and host strains: nucleotide sequences of the M13mp18 and pUC19 vectors. Gene 33, $103-119$

Yarnell, W. S., and Roberts, J. W. (1992) The phage 1 gene $Q$ transcription antiterminator binds DNA in the late gene promoter as it modifies RNA polymerase. Cell 69, 1181-1189.

Yee, A. J., de Grandis, S., and Gyles, C. L. (1993) Mitomycin-induced synthesis of a Shiga-like toxin from enteropathogenic Escherichia coli H. I. 8. Infect. Immun. 61, 4510-4513. 